\title{
PMT gain calibration and monitoring based on highly compressed hit information in KM3NeT
}

\section{Bouke Jung, ${ }^{a, b, *}$ Maarten de Jong ${ }^{a, c}$ and Paolo Fermani ${ }^{d}$ on behalf of the KM3NeT Collaboration}

(a complete list of authors can be found at the end of the proceedings)

${ }^{a}$ Dutch National Institute for Subatomic Physics (Nikhef),

Science Park 105, Amsterdam, The Netherlands

University $b$ of Amsterdam, Institute of Physics,

Science Park 904, Amsterdam, The Netherlands

${ }^{c}$ Leiden University, Leiden Institute of Physics,

Niels Bohrweg 2, Leiden, The Netherlands

${ }^{b}$ Sapienza Università di Roma, Dipartimento di Fisica,

Piazzale Aldo Moro 5, Rome, Italy

E-mail: bjung@nikhef.nl, mjg@nikhef.nl, paolo.fermani@roma1.infn.it

The cubic-kilometre neutrino telescope, consisting of large-scale 3D-arrays of photomultiplier tubes (PMTs) currently under construction on the Mediterranean seabed, relies on accurate calibration procedures in order to answer its science goals. These proceedings present an overview of a novel gain calibration method based on highly compressed PMT hit information. In particular, it is shown that the PMT gains can be tuned to within $2 \%$ of the nominal value, based on the measured time-over-threshold.

$37^{\text {th }}$ International Cosmic Ray Conference (ICRC 2021)

July 12th-23rd, 2021

Online - Berlin, Germany

\footnotetext{
*Presenter
} 


\section{Introduction}

The cubic kilometre neutrino telescope (KM3NeT), currently under construction at two sites on the Mediterranean seabed, consists of two large-volume water Cherenkov detectors, which aim to answer a number of open questions in neutrino physics and astrophysics [1], particularly:

I.) What is the origin of cosmic neutrinos?

II.) Which neutrino mass ordering is realized in nature?

The first question is addressed by the ARCA detector (for Astroparticle Research with Cosmics in the Abyss), situated at an approximate depth of 3500 metres, about 100 kilometres off-shore from the small town of Portopalo di Capo Passero on Sicily, Italy [2]. The second question is investigated using the ORCA detector (for Oscillations Research with Cosmics in the Abyss), located at a depth of approximately 2450 metres, 40 kilometres off-shore from Toulon, in Southern France [3].

Both detectors use a similar infrastructure, consisting of building blocks with 115 strings, which comprise 18 optical modules each. The optical modules are instrumented with 31 Hamamatsu R12199-02 3-inch photo-multiplier tubes (PMTs), which are able to detect individual Cherenkov photons emitted during neutrino interactions in the seawater.

In order to achieve the angular and energy resolutions necessary to pursue the science goals of KM3NeT, accurate calibration is essential. This calibration has to be achieved on the basis of PMT hit information with a high compression rate, which is needed to maximize the fraction of raw data which can be sent to shore for later neutrino event triggering, whilst keeping the $7 \mathrm{kHz}$ background expected from radioactive decays and bioluminescence in the seawater well below the limit of 200 Mbps per optical module, set by the maximum throughput of the deployed fibre-optics [4]. The compression is achieved by reducing the full PMT waveforms corresponding to individual PMT hits to data-packets of 6 Bytes, comprising 1 Byte for storage of the PMT address, 4 Bytes for storage of the hit arrival time and 1 Byte for storage of the time duration during which the PMT analogue pulse exceeded the threshold set by the hardware discriminator. Previous papers have already described methods for the calibration of the PMT hit arrival times and quantum efficiencies based on this highly compressed PMT hit information [5-7]. In contrast, these proceedings present an overview of the methods which allow for the monitoring and calibration of the gain of the PMTs inside KM3NeT.

\section{The PMT analogue pulse model}

The primary output of a PMT consists of an electrical current, also referred to as an analogue pulse, which is read out at the anode. These currents are generated when primary electrons, created by photons impinging on the photocathode, are accelerated through the PMT's dynode system. The dynode system consists of an array of metal wafers, each with a secondary emission coefficient greater than one, such that encounters with successive dynodes lead to a multiplication of the outgoing current. The average amplification factor, measured over an ensemble of PMT hits associated 
with the release of single primary electrons at the photocathode (i.e. single photoelectron hits), is referred to as the gain.

Typically, the gain of a PMT is evaluated by integrating a large number of waveforms over time and fitting the mean and variance of the resulting charge distribution to a Gaussian [8]. This is not possible for KM3NeT, because neither the full waveforms nor the integral are stored. Instead, the gain needs to be inferred from the statistical behaviour of the time-over-threshold. This is done using an analytical model which converts measured time-over-threshold values into the charge of the underlying pulse and vice versa.

To good approximation the analogue pulses measured at the anode of a PMT can be modelled as a Gaussian with an exponential tail. The resulting output voltage can therefore be expressed as follows in terms of the time $t$ and the charge $q$, associated with the pulse:

$$
V(t, q)= \begin{cases}q R e^{-\frac{1}{2}\left(\frac{t}{\sigma}\right)^{2}}, & t \leq \tilde{t}, \\ \frac{q R}{C} e^{\frac{-t}{\tau}}, & t>\tilde{t} .\end{cases}
$$

In this equation, $\sigma$ is the width of the Gaussian component of the pulse and $\tau$ is the decay-time associated with the exponential part, whereas $C$ and $R$ are two normalisation constants. The latter constant has units of resistance over time and is related to the electronic circuit's load resistance, whilst the former can be defined as the normalized pulse height evaluated at the time, $\tilde{t}=\frac{\sigma^{2}}{\tau}$, where the Gaussian and exponential part of the pulse shape match both in terms of their amplitudes and derivatives, i.e. $C \equiv \frac{V(\tilde{t}, q)}{q R}=e^{-\frac{1}{2}\left(\frac{\sigma}{\tau}\right)^{2}}$.

By inverting equation 1 an expression can be derived for the time-over-threshold in terms of the charge associated with the analogue pulse. Assuming the hardware discriminator sets a voltage threshold equal to $V_{0}$, the leading and trailing edge of the analogue pulse, defined as the first and, respectively, last time at which the pulse exceeds the threshold, can be defined as:

$$
\begin{aligned}
& t_{1}=-\sigma \sqrt{2 \ln \left(\frac{q R}{V_{0}}\right),} \\
& t_{2}= \begin{cases}\sigma \sqrt{2 \ln \left(\frac{q R}{V_{0}}\right),} & V(\tilde{t}, q) \leq V_{0}, \\
\tau \ln \left(\frac{q R}{C V_{0}}\right), & V(\tilde{t}, q)>V_{0} .\end{cases}
\end{aligned}
$$

The time-over-threshold is computed as the difference between these times, with the addition of two phenomenological modifications. The first modification accounts for the effect of clipping, where the top part of pulses with an amplitude in excess of the voltage bias effectively get cut off. This results in a linear extension of the time-over-threshold with additional charge [9]. The second modification accounts for the effect of saturation, caused by the read-out electronics. Studies performed in the lab have shown that this effect can be approximated well using an inverse square root modulation, limiting the time-over-threshold to a maximum value, $\Delta T_{\max }$. 


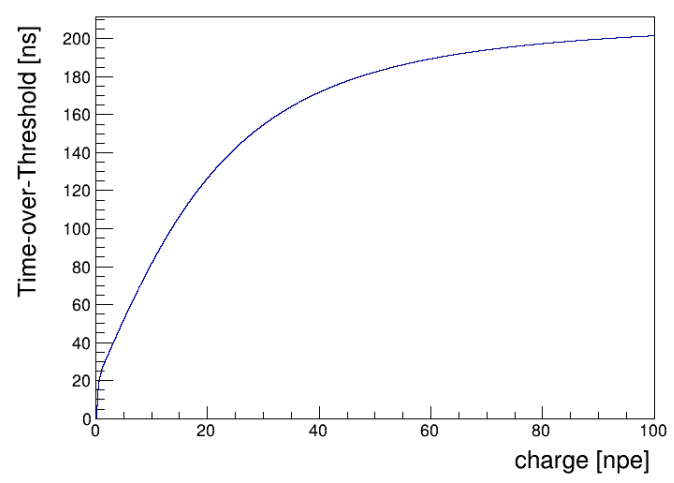

Figure 1: The time-over-threshold as function of the charge, as described by equation 4 .

Summarizing all of the above, the time-over-threshold corresponding to an analogue pulse with total charge, $q$, can be calculated as:

$$
\Delta T=\frac{\Delta T_{\max }}{\sqrt{\Delta T_{\max }^{2}+\Delta \tilde{T}^{2}(q)}} \cdot \Delta \tilde{T}(q),
$$

where $\Delta \tilde{T}(q)$ is given by:

$$
\Delta \tilde{T}(q)= \begin{cases}2 \sigma \sqrt{2 \ln \left(\frac{q}{q_{0}}\right)}, & q_{0}<q \leq \frac{q_{0}}{C}, \\ \tau \ln \left(\frac{q}{q_{0} \cdot C}\right)+\sigma \sqrt{2 \ln \left(\frac{q}{q_{0}}\right)}, & \frac{q_{0}}{C}<q \leq q_{\mathrm{L}}, \\ \tau \ln \left(\frac{q_{\mathrm{L}}}{q_{0} \cdot C}\right)+\sigma \sqrt{2 \ln \left(\frac{q_{\mathrm{L}}}{q_{0}}\right)}+\beta\left(q-q_{\mathrm{L}}\right), & q>q_{\mathrm{L}}, \\ 0, & q \leq q_{0} .\end{cases}
$$

In this relation, $q_{0}$ is the threshold-equivalent charge, set by the condition $V\left(0, q_{0}\right)=V_{0}$ and $\beta$ is the derivative of the time-over-threshold with respect to the charge in the linear regime corresponding to clipped analogue pulses. Given the nominal signal amplification factor of $3 \cdot 10^{6}[10]$, the value of $\beta$ has been determined experimentally to be 7.0 ns p.e. $^{-1}$ [11]. The lower bound of the linear regime, $q_{\mathrm{L}}$, can be chosen as the point where the derivative of equation 5 becomes equal to this experimentally established value of $\beta$. This point can be typically found around a charge equivalent to 1 p.e..

Equations 4 and 5 show that the dependency between the time-over-threshold and the charge of the analogue pulse can be divided up into three regimes: one regime for pulses with low amplitude with a trailing edge in the Gaussian domain of the pulse shape, one regime for pulses with a trailing edge in the exponential tail and one regime corresponding to pulses with high amplitude which are clipped. The full functional behaviour of equation 4 is shown in figure 1 . 


\section{The charge distribution}

To use the time-over-threshold as a measure for the PMT gain, the shape of the charge distribution for single photoelectron PMT hits must be defined first. One charge distribution model which is often used in the characterizations of PMTs because of its simplicity, is a single Gaussian, centered around the PMT gain [8]. Such models have however been reported to underestimate the lower end of the single photoelectron charge distribution, where contributions from underamplified signals and dark counts can be high [12,13]. To account for these contributions, the single photoelectron charge distribution in KM3NeT is instead modelled as a two-component Gaussian mixture:

$$
f(q)=\frac{1}{A}\left(p \cdot \mathcal{G}\left(q ; \mu_{u}, \sigma_{u}^{2}\right)+(1-p) \cdot \mathcal{G}\left(q ; \mathrm{G}, \Sigma^{2}\right)\right)
$$

where $A$ is a normalisation constant which accounts for the truncation of the distribution by the hardware threshold, $p$ corresponds to the occurrence probability of an underamplified hit and $\mathcal{G}\left(x ; \mu, \sigma^{2}\right)$ corresponds to a Gaussian distribution with mean value $\mu$ and standard deviation $\sigma$. The mean and standard deviation of the nominal (underamplified) distribution are referred to as $\mathrm{G}$ and $\Sigma\left(\mu_{u}\right.$ and $\left.\sigma_{u}\right)$, respectively. They are related by the expression $\Sigma=\sigma_{\mathrm{G}} \sqrt{\mathrm{G}}$, where $\mathrm{G}$ and $\sigma_{\mathrm{G}}$ correspond to the gain and the gainspread of the PMT. Assuming that the dynode responses are Poissonian and that the final charge distribution is caused by statistical fluctuations mostly in the first electron multiplication step, the mean and standard deviation of the underamplified Gaussian can be assumed approximately equal to $\mu_{u}=\sigma_{G}^{2} \cdot G=\Sigma^{2}$ and $\mu_{u}=\sigma_{G} \cdot \Sigma$. Effectively this means that a shift of the nominally amplified distribution to the right or the left will lead to a similar shift in the underamplified distribution. Figure 2 shows the model for the single-photo electron charge distribution between 0.0 p.e. and 2.0 p.e.. The truncation by the threshold can be seen around 0.12 p.e..
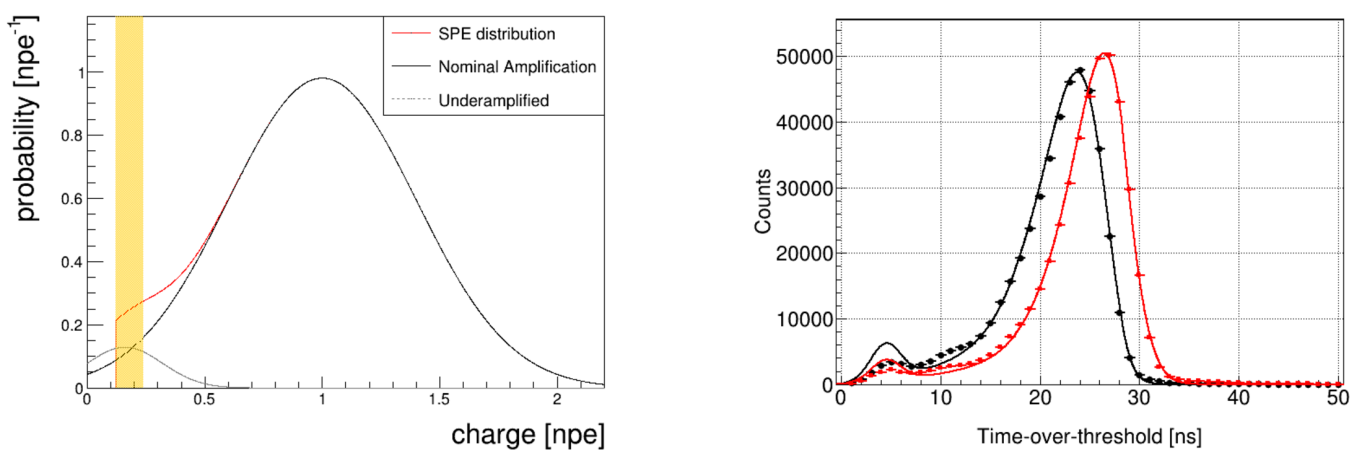

Figure 2: The single photoelectron charge distribution model given by equation 6 for $p=0.05$ and $\sigma_{\mathrm{G}}=0.3$ (left) and two time-over-threshold distributions taken for a PMT operated at the ORCA site under high voltages of $-1130 \mathrm{~V}$ (black) and $-1180 \mathrm{~V}$ (red), fitted according to equation 8 (right). Individual contributions from underamplified and nominal signals are indicated in the left figure with black dotted and solid lines, respectively. The area marked in orange shows the threshold band. 


\section{The Time-over-Threshold distribution and PMT gain calibration}

Equations 4 and 6 provide a definition of the time-over-threshold, given by:

$$
f(\Delta T)=f(q(\Delta T)) \cdot \frac{\partial q(\Delta T)}{\partial \Delta T} .
$$

This model fits the main component of the time-over-threshold distribution well, but does not fully capture the lower end of the distribution. In particular, it underestimates the region around $4.5 \mathrm{~ns}$, which contains a small bump originating from real, stand-alone photon hits, with small amplitudes surrounding the voltage threshold. This component is accounted for phenomenologically by adding a Gaussian contribution, which scales according to the fraction of the charge distribution inside a small region below the approximate threshold-equivalent-charge. The region, referred to as the threshold band, has a fixed width given by the parameter, $q_{b}$, and can be seen on the left-hand side of figure 2 as an orange band. Including it, the time-over-threshold distribution can be defined as:

$$
f(\Delta T)=W_{\mathrm{a}} \cdot f(q(\Delta T)) \cdot \frac{\partial q(\Delta T)}{\partial \Delta T}+W_{b} \cdot \mathcal{G}\left(\Delta T ; \mu_{n}, \sigma_{n}\right),
$$

where $\mu_{n} \approx 4.5 \mathrm{~ns}$ and $\sigma_{n} \approx 1.5 \mathrm{~ns}$ denote the mean and standard deviation of the observed excess. The normalisation constants $W_{a}$ and $W_{b}$ are defined as $W_{b}=\int_{q_{0}-q_{b}}^{q_{0}} f(q) d q$ and $W_{\mathrm{a}}=1-W_{\mathrm{b}}=\int_{q_{0}}^{\infty} f(q) d q$.

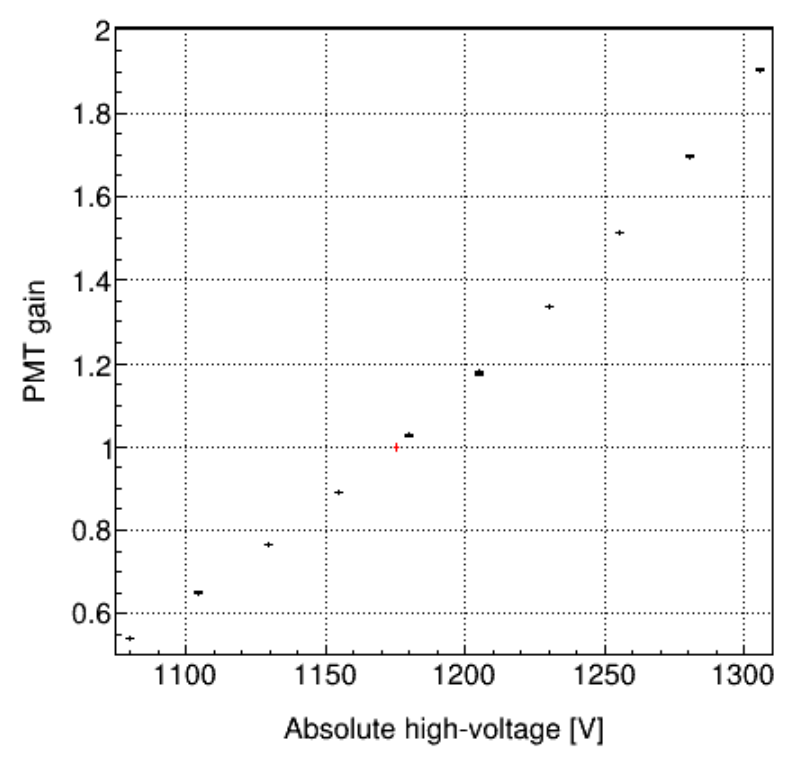

Figure 3: Fitted gain values normalized with respect to the nominal amplification factor of $3 \cdot 10^{6}$ as a function of the applied absolute high voltage. The nominal gain value, indicated as a red point, is interpolated linearly on a $\log -\log$ scale. 
Equation 8 relates the single photoelectron time-over-threshold distribution to the gain and gainspread of the underlying charge distribution given by equation 6 . This relation can be exploited for the purpose of gain monitoring and calibration. On the right-hand side of figure 2, two timeover-threshold distributions are shown, which have been acquired for different settings of the high voltage. Both distributions have been fitted as a function of the gain and the gainspread of the single photoelectron charge distribution. By repeating fits like these over a range of applied high voltages, the nominal gain, corresponding to an amplification factor of $3 \cdot 10^{6}$, can be interpolated. This is shown in figure 3. Because the gain and high voltage are related approximately as a power-law [14], the resulting datapoints are put on a logarithmic $x$ - and y-scale first, before proceeding with the interpolation.

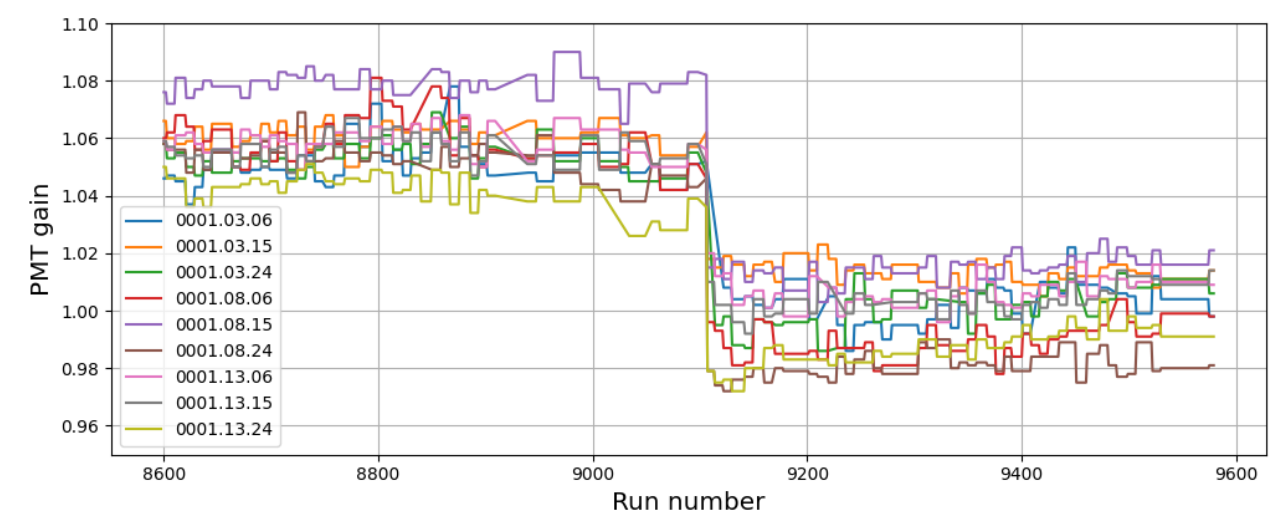

Figure 4: The gains for a selection of 9 PMTs monitored in a string deployed at the ORCA site over a timespan of approximately half a year, between September 2020 and March 2021 (1 run corresponds roughly to 3 hours). A gain-calibration campaign is visible as a discontinuous jump after run 9100.

Figure 4 shows the evolution of the normalized gain for a selection of 9 different PMTs in a string deployed at the ORCA site, monitored between September 2020 and March 2021. As can be seen, a gain calibration campaign undertaken in December 2019 successfully brought the PMT gains back to a value within around $2 \%$ of the nominal value of 1.0 , defined as a fraction of the nominal amplification factor of $3 \cdot 10^{6}$.

\section{Conclusions}

In these proceedings, an outline has been given of the procedure which is used to calibrate the gains of the KM3NeT PMTs, based on hit information with a high compression rate. In particular, an analytical model has been described which allows for the conversion of the single Byte time-overthreshold information recorded for each PMT hit, to the integrated charge under the corresponding analogue pulse and vice versa. This model allows measured time-over-threshold distributions for single photoelectron hits to be fitted in terms of the gain on a PMT-by-PMT basis.

In addition, it has been shown how the PMT gains can be calibrated by fitting time-overthreshold distributions over a range of high-voltage settings and interpolating the high voltage 
which yields a nominal gain, corresponding to an amplification factor of $3 \cdot 10^{6}$. Recent calibration campaigns shown in these proceedings, indicate that this method allows the gain to be tuned to within $2 \%$ of the nominal value.

\section{References}

[1] Annarita Margiotta. The km3net deep-sea neutrino telescope. Nuclear Instruments and Methods in Physics Research Section A: Accelerators, Spectrometers, Detectors and Associated Equipment, 766:83-87, 2014. RICH2013 Proceedings of the Eighth International Workshop on Ring Imaging Cherenkov Detectors Shonan, Kanagawa, Japan, December 2-6, 2013.

[2] S. Aiello, S.E. Akrame, F. Ameli, et al. Sensitivity of the KM3NeT/ARCA neutrino telescope to point-like neutrino sources. Astroparticle Physics, 111:100-110, 2019.

[3] S. Aiello, A. Albert, S. Alves Garre, et al. Determining the Neutrino Mass Ordering and Oscillation Parameters with KM3NeT/ORCA, 2021.

[4] Simone Biagi et al. The data acquisition system of the KM3NeT detector. PoS (ICRC2015), 1172, 2015.

[5] Silvia Adrián-Martínez, M Ageron, F Aharonian, et al. The prototype detection unit of the KM3NeT detector. The European Physical Journal C, 76(2):1-12, 2016.

[6] KW Melis. In-situ calibration of KM3NeT. PoS (ICRC2017), 1059:123, 2017.

[7] Rosa Coniglione, Alexandre Creusot, Irene Di Palma, et al. KM3NeT time calibration. In 36th International Cosmic Ray Conference, volume 358, page 868. SISSA Medialab, 2019.

[8] E.H. Bellamy, G. Bellettini, J. Budagov, et al. Absolute calibration and monitoring of a spectrometric channel using a photomultiplier. Nuclear Instruments and Methods in Physics Research Section A: Accelerators, Spectrometers, Detectors and Associated Equipment, 339(3):468 - 476, 1994.

[9] Jonas Reubelt. Hardware studies, in-situ prototype calibration and data analysis of the novel multi-PMT digital optical module for the KM3NeT neutrino telescope. 2019.

[10] S. Aiello, S.E. Akrame, F. Ameli, et al. Characterisation of the Hamamatsu photomultipliers for the KM3NeT Neutrino Telescope. Journal of Instrumentation, 13(05):P05035-P05035, may 2018.

[11] A.S. Schermer. Characterizing the KM3NeT 3-inch Hamamatsu Photomultiplier Tube response. Master's thesis, University of Amsterdam, Science Park 105, 1098 XG Amsterdam, The Netherlands, 2017.

[12] J.R. Prescott. A statistical model for photomultiplier single-electron statistics. Nuclear Instruments and Methods, 39(1):173 - 179, 1966.

[13] Pavel Degtiarenko. Precision analysis of the photomultiplier response to ultra low signals. Nuclear Instruments and Methods in Physics Research Section A: Accelerators, Spectrometers, Detectors and Associated Equipment, 872:1-15, Nov 2017.

[14] AG Wright. The photomultiplier handbook. Oxford University Press, 2017. 


\section{Full Authors List: KM3NeT Collaboration}

M. Ageron ${ }^{1}$, S. Aiello ${ }^{2}$, A. Albert ${ }^{3,55}$, M. Alshamsi ${ }^{4}$, S. Alves Garre $^{5}$, Z. Aly ${ }^{1}$, A. Ambrosone $^{6,7}$, F. Ameli ${ }^{8}$, M. Andre $^{9}$, G. Androulakis $^{10}$, M. Anghinolfi ${ }^{11}$, M. Anguita ${ }^{12}$, G. Anton ${ }^{13}$, M. Ardid ${ }^{14}, S$. Ardid $^{14}$,W. Assal ${ }^{1}$, J. Aublin ${ }^{4}$, C. Bagatelas ${ }^{10}$, B. Baret ${ }^{4}$, S. Basegmez du Pree ${ }^{15}$, M. Bendahman ${ }^{4,16}$, F. Benfenati ${ }^{17,18}$, E. Berbee ${ }^{15}$, A. M. van den Berg ${ }^{19}$, V. Bertin ${ }^{1}$, S. Beurthey ${ }^{1}$, V. van Beveren ${ }^{15}$, S. Biagi ${ }^{20}$, M. Billault ${ }^{1}$, M. Bissinger ${ }^{13}$, M. Boettcher ${ }^{21}$, M. Bou Cabo $^{22}$, J. Boumaaza ${ }^{16}$, M. Bouta ${ }^{23}$, C. Boutonnet ${ }^{4}$, G. Bouvet ${ }^{24}$, M. Bouwhuis ${ }^{15}$, C. Bozza ${ }^{25}$, H.Brânzaş ${ }^{26}$, R. Bruijn ${ }^{15,27}$, J. Brunner ${ }^{1}$, R. Bruno ${ }^{2}$, E. Buis ${ }^{28}$, R. Buompane ${ }^{6,29}$, J. Busto ${ }^{1}$, B. Caiff ${ }^{11}$,

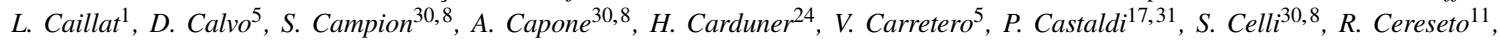

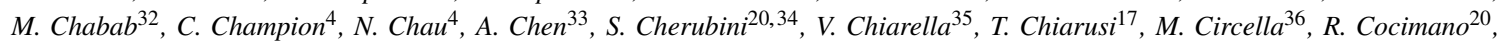
J. A. B. Coelho ${ }^{4}$, A. Coleiro ${ }^{4}$, M. Colomer Molla ${ }^{4,5}$, S. Colonges ${ }^{4}$, R. Coniglione ${ }^{20}$, A. Cosquer ${ }^{1}$, P. Coyle ${ }^{1}$, M. Cresta ${ }^{11}$, A. Creusot ${ }^{4}$, A. Cruz ${ }^{37}$, G. Cuttone ${ }^{20}$, A. D'Amico ${ }^{15}$, R. Dallier ${ }^{24}$, B. De Martino ${ }^{1}$, M. De Palma ${ }^{36,38}$, I. Di Palma ${ }^{30,8}$, A. F. Díaz ${ }^{12}$, D. DiegoTortosa $^{14}$, C. Distefano ${ }^{20}$, A. Domi ${ }^{15,27}$, C. Donzaud ${ }^{4}$, D. Dornic ${ }^{1}$, M. Dörr ${ }^{39}$, D. Drouhin ${ }^{3,55}$, T. Eberl ${ }^{13}$, A. Eddyamoui ${ }^{16}$, T. van Eeden ${ }^{15}$, D. van Eijk ${ }^{15}$, I. El Bojaddaini ${ }^{23}$, H. Eljarrari $^{16}$, D. Elsaesser ${ }^{39}$, A. Enzenhöfer $^{1}$, V. Espinosa ${ }^{14}$, P. Fermani $^{30,8}$, G. Ferrara ${ }^{20,34}$, M. D. Filipovic ${ }^{40}$, F. Filippini ${ }^{17,18}$, J. Fransen ${ }^{15}$, L. A. Fusco ${ }^{1}$, D. Gajanana ${ }^{15}$, T. Gal ${ }^{13}$, J. García Méndez ${ }^{14}$, A. Garcia Soto $^{5}$, E. Garçon ${ }^{1}$, F. Garufi ${ }^{6,7}$, C. Gatius ${ }^{15}$, N. Geißelbrecht ${ }^{13}$, L. Gialanella ${ }^{6,29}$, E. Giorgio ${ }^{20}$, S. R. Gozzini ${ }^{5}$, R. Gracia ${ }^{15}$, K. Graf ${ }^{13}$, G. Grella ${ }^{41}$, D. Guderian ${ }^{56}$, C. Guidi ${ }^{11,42}$, B. Guillon ${ }^{43}$, M. Gutiérrez $^{44}$, J. Haefner ${ }^{13}$, S. Hallmann ${ }^{13}$, H. Hamdaoui $^{16}$, H. van Haren ${ }^{45}$, A. Heijboer ${ }^{15}$, A. Hekalo ${ }^{39}$, L. Hennig ${ }^{13}$, S. Henry ${ }^{1}$, J. J. Hernández-Rey ${ }^{5}$, J. Hofestädt ${ }^{13}$, F. Huang ${ }^{1}$, W. Idrissi Ibnsalih ${ }^{6,29}$, A. Ilioni ${ }^{4}$, G. Illuminati ${ }^{17,18,4}$, C.W. James ${ }^{37}$, D. Janezashvili ${ }^{46}$, P. Jansweijer ${ }^{15}$, M. de Jong ${ }^{15,47}$, P. de Jong ${ }^{15,27}$, B. J. Jung ${ }^{15}$, M. Kadler ${ }^{39}$, P. Kalaczyński ${ }^{48}$, O. Kalekin ${ }^{13}$, U. F. Katz ${ }^{13}$, F. Kayzel ${ }^{15}$, P. Keller ${ }^{1}$, N. R. Khan Chowdhury ${ }^{5}$, G. Kistauri ${ }^{46}$, F. van der Knaap ${ }^{28}$, P. Kooijman ${ }^{27,57}$, A. Kouchner ${ }^{4,49}$, M. Kreter ${ }^{21}$, V. Kulikovskiy ${ }^{11}$, M. Labalme ${ }^{43}$, P. Lagier $^{1}$, R. Lahmann ${ }^{13}$, P. Lamare ${ }^{1}$, M. Lamoureux $^{14}$, G. Larosa ${ }^{20}$, C. Lastoria ${ }^{1}$, J. Laurence ${ }^{1}$, A. Lazo ${ }^{5}$, R. Le Breton ${ }^{4}$, E. Le Guirriec ${ }^{1}$, S. Le Stum ${ }^{1}$, G. Lehaut ${ }^{43}$, O. Leonardi ${ }^{20}$, F. Leone ${ }^{20,34}$, E. Leonora ${ }^{2}$, C. Lerouvillois ${ }^{1}$, J. Lesrel $^{4}$, N. Lessing ${ }^{13}$, G. Levi ${ }^{17,18}$, M. Lincetto $^{1}$, M. Lindsey Clark ${ }^{4}$, T. Lipreau ${ }^{24}$, C. LLorens Alvarez ${ }^{14}$, A. Lonardo ${ }^{8}$, F. Longhitano ${ }^{2}$, D. Lopez-Coto ${ }^{44}$, N. Lumb ${ }^{1}$, L. Maderer ${ }^{4}$, J. Majumdar ${ }^{15}$, J. Mańczak ${ }^{5}$, A. Margiotta ${ }^{17,18}$, A. Marinelli ${ }^{6}$, A. Marini ${ }^{1}$, C. Markou ${ }^{10}$, L. Martin ${ }^{24}$, J.A. Martínez-Mora ${ }^{14}$, A. Martini ${ }^{35}$, F. Marzaioli ${ }^{6,29}$,

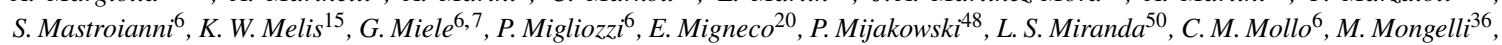
A. Moussa ${ }^{23}$, R. Muller ${ }^{15}$, P. Musico ${ }^{11}$, M. Musumeci ${ }^{20}$, L. Nauta ${ }^{15}$, S. Navas ${ }^{44}$, C.A. Nicolau ${ }^{8}$, B. Nkosi ${ }^{33}$, B. Ó Fearraigh ${ }^{15,27}$, M. O'Sullivan ${ }^{37}$, A. Orlando ${ }^{20}$, G. Ottonello ${ }^{11}$, S. Ottonello ${ }^{11}$, J. Palacios González ${ }^{5}$, G. Papalashvilit ${ }^{46}$, R. Papaleo ${ }^{20}$, C. Pastore ${ }^{36}$,

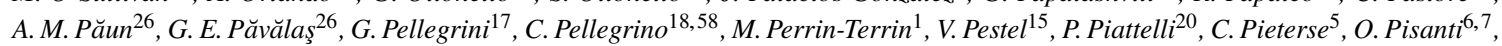
C. Poirè ${ }^{14}$, V. Popa ${ }^{26}$, T. Pradier ${ }^{3}$, F. Pratolongo ${ }^{11}$, I. Probst ${ }^{13}$, G. Pühlhofer ${ }^{51}$, S. Pulvirenti ${ }^{20}$, G. Quéméner ${ }^{43}$, N. Randazzo ${ }^{2}$, A. Rapicavoli ${ }^{34}$, S. Razzaque ${ }^{50}$, D. Real ${ }^{5}$, S. Reck ${ }^{13}$, G. Riccobene ${ }^{20}$, L. Rigalleau ${ }^{24}$, A. Romanov ${ }^{11,42}$, A. Rovelli ${ }^{20}$, J. Royon ${ }^{1}$, F. Salesa Greus ${ }^{5}$, D. F. E. Samtleben ${ }^{15,47}$, A. Sánchez Losa ${ }^{36,5}$, M. Sanguineti ${ }^{11,42}$, A. Santangelo ${ }^{51}$, D. Santonocito ${ }^{20}$, P. Sapienza ${ }^{20}$, J. Schmelling ${ }^{15}$, J. Schnabel ${ }^{13}$, M. F. Schneider ${ }^{13}$, J. Schumann ${ }^{13}$, H. M. Schutte ${ }^{21}$, J. Seneca ${ }^{15}$, I. Sgura ${ }^{36}$, R. Shanidze 46 $^{46}$. Sharma ${ }^{52}$, A. Sinopoulou ${ }^{10}$, B. Spisso ${ }^{41,6}$, M. Spurio ${ }^{17,18}$, D. Stavropoulos ${ }^{10}$, J. Steijger ${ }^{15}$, S. M. Stellacci ${ }^{41,6}$, M. Taiuti ${ }^{11,42}$, F. Tatone ${ }^{36}$, Y. Tayalati ${ }^{16}$, E. Tenllado ${ }^{44}$, D. Tézier ${ }^{1}$, T. Thakore ${ }^{5}$, S. Theraube ${ }^{1}$, H. Thiersen ${ }^{21}$, P. Timmer ${ }^{15}$, S. Tingay ${ }^{37}$, S. Tsagkli ${ }^{10}$, V. Tsourapis ${ }^{10}$, E. Tzamariudaki ${ }^{10}$, D. Tzanetatos $^{10}$, C. Valieri ${ }^{17}$, V. Van Elewyck ${ }^{4,49}$, G. Vasileiadis ${ }^{53}$, F. Versari ${ }^{17,18}$, S. Viola ${ }^{20}$, D. Vivolo ${ }^{6,29}$, G. de Wasseige ${ }^{4}$, J. Wilms ${ }^{54}$, R. Wojaczyński ${ }^{48}$, E. de Wolf ${ }^{15,27}$, T. Yousfi $^{23}$, S. Zavatarelli ${ }^{11}$, A. Zegarelli ${ }^{30,8}$, D. Zito ${ }^{20}$, J. D. Zornoza ${ }^{5}$, J. Zúñiga ${ }^{5}, N$. Zywucka ${ }^{21}$.

${ }^{1}$ Aix Marseille Univ, CNRS/IN2P3, CPPM, Marseille, France.

${ }^{2}$ INFN, Sezione di Catania, Via Santa Sofia 64, Catania, 95123 Italy.

${ }^{3}$ Université de Strasbourg, CNRS, IPHC UMR 7178, F-67000 Strasbourg, France.

${ }^{4}$ Université de Paris, CNRS, Astroparticule et Cosmologie, F-75013 Paris, France.

${ }^{5}$ IFIC - Instituto de Física Corpuscular (CSIC - Universitat de València), c/Catedrático José Beltrán, 2, 46980 Paterna, Valencia, Spain.

${ }^{6}$ INFN, Sezione di Napoli, Complesso Universitario di Monte S. Angelo, Via Cintia ed. G, Napoli, 80126 Italy.

${ }^{7}$ Università di Napoli "Federico II", Dip. Scienze Fisiche "E. Pancini”, Complesso Universitario di Monte S. Angelo, Via Cintia ed. G, Napoli, 80126 Italy.

${ }^{8}$ INFN, Sezione di Roma, Piazzale Aldo Moro 2, Roma, 00185 Italy.

${ }^{9}$ Universitat Politècnica de Catalunya, Laboratori d'Aplicacions Bioacústiques, Centre Tecnològic de Vilanova i la Geltrú, Avda. Rambla Exposició, s/n, Vilanova i la Geltrú, 08800 Spain.

${ }^{10}$ NCSR Demokritos, Institute of Nuclear and Particle Physics, Ag. Paraskevi Attikis, Athens, 15310 Greece.

${ }^{11}$ INFN, Sezione di Genova, Via Dodecaneso 33, Genova, 16146 Italy.

${ }^{12}$ University of Granada, Dept. of Computer Architecture and Technology/CITIC, 18071 Granada, Spain.

${ }^{13}$ Friedrich-Alexander-Universität Erlangen-Nürnberg, Erlangen Centre for Astroparticle Physics, Erwin-Rommel-Straße 1, 91058 Erlangen, Germany.

${ }^{14}$ Universitat Politècnica de València, Instituto de Investigación para la Gestión Integrada de las Zonas Costeras, C/ Paranimf, 1, Gandia, 46730 Spain.

${ }^{15}$ Nikhef, National Institute for Subatomic Physics, PO Box 41882, Amsterdam, 1009 DB Netherlands.

${ }^{16}$ University Mohammed V in Rabat, Faculty of Sciences, 4 av. Ibn Battouta, B.P. 1014, R.P. 10000 Rabat, Morocco.

\footnotetext{
${ }^{1}$ also at Dipartimento di Fisica, INFN Sezione di Padova and Università di Padova, I-35131, Padova, Italy
} 
${ }^{17}$ INFN, Sezione di Bologna, v.le C. Berti-Pichat, 6/2, Bologna, 40127 Italy.

${ }^{18}$ Università di Bologna, Dipartimento di Fisica e Astronomia, v.le C. Berti-Pichat, 6/2, Bologna, 40127 Italy.

${ }^{19}$ KVI-CART University of Groningen, Groningen, the Netherlands.

${ }^{20}$ INFN, Laboratori Nazionali del Sud, Via S. Sofia 62, Catania, 95123 Italy.

${ }^{21}$ North-West University, Centre for Space Research, Private Bag X6001, Potchefstroom, 2520 South Africa.

${ }^{22}$ Instituto Español de Oceanografía, Unidad Mixta IEO-UPV, C/ Paranimf, 1, Gandia, 46730 Spain.

${ }^{23}$ University Mohammed I, Faculty of Sciences, BV Mohammed VI, B.P. 717, R.P. 60000 Oujda, Morocco.

${ }^{24}$ Subatech, IMT Atlantique, IN2P3-CNRS, Université de Nantes, 4 rue Alfred Kastler - La Chantrerie, Nantes, BP 2072244307 France.

${ }^{25}$ Università di Salerno e INFN Gruppo Collegato di Salerno, Dipartimento di Matematica, Via Giovanni Paolo II 132, Fisciano, 84084 Italy.

${ }^{26}$ ISS, Atomistilor 409, Măgurele, RO-077125 Romania.

${ }^{27}$ University of Amsterdam, Institute of Physics/IHEF, PO Box 94216, Amsterdam, 1090 GE Netherlands.

${ }^{28}$ TNO, Technical Sciences, PO Box 155, Delft, 2600 AD Netherlands.

${ }^{29}$ Università degli Studi della Campania "Luigi Vanvitelli", Dipartimento di Matematica e Fisica, viale Lincoln 5, Caserta, 81100 Italy.

${ }^{30}$ Università La Sapienza, Dipartimento di Fisica, Piazzale Aldo Moro 2, Roma, 00185 Italy.

${ }^{31}$ Università di Bologna, Dipartimento di Ingegneria dell'Energia Elettrica e dell'Informazione "Guglielmo Marconi", Via dell'Università 50, Cesena, 47521 Italia.

${ }^{32}$ Cadi Ayyad University, Physics Department, Faculty of Science Semlalia, Av. My Abdellah, P.O.B. 2390, Marrakech, 40000 Morocco.

${ }^{33}$ University of the Witwatersrand, School of Physics, Private Bag 3, Johannesburg, Wits 2050 South Africa.

${ }^{34}$ Università di Catania, Dipartimento di Fisica e Astronomia "Ettore Majorana", Via Santa Sofia 64, Catania, 95123 Italy.

${ }^{35}$ INFN, LNF, Via Enrico Fermi, 40, Frascati, 00044 Italy.

${ }^{36}$ INFN, Sezione di Bari, via Orabona, 4, Bari, 70125 Italy.

${ }^{37}$ International Centre for Radio Astronomy Research, Curtin University, Bentley, WA 6102, Australia.

${ }^{38}$ University of Bari, Via Amendola 173, Bari, 70126 Italy.

${ }^{39}$ University Würzburg, Emil-Fischer-Straße 31, Würzburg, 97074 Germany.

${ }^{40}$ Western Sydney University, School of Computing, Engineering and Mathematics, Locked Bag 1797, Penrith, NSW 2751 Australia.

${ }^{41}$ Università di Salerno e INFN Gruppo Collegato di Salerno, Dipartimento di Fisica, Via Giovanni Paolo II 132, Fisciano, 84084 Italy.

${ }^{42}$ Università di Genova, Via Dodecaneso 33, Genova, 16146 Italy.

${ }^{43}$ Normandie Univ, ENSICAEN, UNICAEN, CNRS/IN2P3, LPC Caen, LPCCAEN, 6 boulevard Maréchal Juin, Caen, 14050 France.

${ }^{44}$ University of Granada, Dpto. de Física Teórica y del Cosmos \& C.A.F.P.E., 18071 Granada, Spain.

${ }^{45}$ NIOZ (Royal Netherlands Institute for Sea Research), PO Box 59, Den Burg, Texel, 1790 AB, the Netherlands.

${ }^{46}$ Tbilisi State University, Department of Physics, 3, Chavchavadze Ave., Tbilisi, 0179 Georgia.

${ }^{47}$ Leiden University, Leiden Institute of Physics, PO Box 9504, Leiden, 2300 RA Netherlands.

${ }^{48}$ National Centre for Nuclear Research, 02-093 Warsaw, Poland.

${ }^{49}$ Institut Universitaire de France, 1 rue Descartes, Paris, 75005 France.

${ }^{50}$ University of Johannesburg, Department Physics, PO Box 524, Auckland Park, 2006 South Africa.

${ }^{51}$ Eberhard Karls Universität Tübingen, Institut für Astronomie und Astrophysik, Sand 1, Tübingen, 72076 Germany.

${ }^{52}$ Università di Pisa, Dipartimento di Fisica, Largo Bruno Pontecorvo 3, Pisa, 56127 Italy.

${ }^{53}$ Laboratoire Univers et Particules de Montpellier, Place Eugène Bataillon - CC 72, Montpellier Cédex 05, 34095 France.

${ }^{54}$ Friedrich-Alexander-Universität Erlangen-Nürnberg, Remeis Sternwarte, Sternwartstraße 7, 96049 Bamberg, Germany.

${ }^{55}$ Université de Haute Alsace, 68100 Mulhouse Cedex, France.

${ }^{56}$ University of Münster, Institut für Kernphysik, Wilhelm-Klemm-Str. 9, Münster, 48149 Germany.

${ }^{57}$ Utrecht University, Department of Physics and Astronomy, PO Box 80000, Utrecht, 3508 TA Netherlands.

${ }^{58}$ INFN, CNAF, v.le C. Berti-Pichat, 6/2, Bologna, 40127 Italy. 